\title{
Leptin in depression: a potential therapeutic target
}

\author{
Tongtong Ge1, Jie Fan', Wei Yang ${ }^{1}$, Ranji Cui ${ }^{1}{ }^{1}$ and Bingjin $\mathrm{Li}^{1}$
}

\begin{abstract}
Leptin, produced and secreted by white adipose tissue, plays a critical role in regulating body weight, food intake, and energy metabolism. Recently, several studies have identified an underlying role for leptin in regulation of mood and cognition via regulation of synaptic changes in the brain that have been associated with antidepressant-like actions. Brain neural plasticity occurs in response to a range of intrinsic and extrinsic stimuli, including those that may mediate the effects of antidepressants. Neural plasticity theories of depression are thought to explain multiple aspects of depression and the effects of antidepressants. It is also well documented that leptin has effects on neural plasticity. This review summarizes the recent literature on the role of leptin in neural plasticity in order to elaborate the possible mechanism of leptin's antidepressant-like effects. Recent findings provide new insights into the underlying mechanisms of neural plasticity in depression. Leptin may influence these mechanisms and consequently constitute a possible target for novel therapeutic approaches to the treatment of depression.
\end{abstract}

\section{Facts}

- Leptin, produced and secreted by white adipose tissue, is a member of the class of hormones called adipokines.

- Leptin can affect mood and cognition via inducing structural and functional alterations in hippocampus and prefrontal cortex.

- Neural plasticity theories of depression may explain multiple aspects of brain plasticity changes associated with depression and antidepressant-like effects.

\section{Open questions}

- Can the decrement of peripheral leptin during the development of depression constitute a biomarker for clinical diagnosis?

\footnotetext{
Correspondence: Ranji Cui (cuiranji@jlu.edu.cn) or Bingjin Li (libingjin@jlu.edu. $\mathrm{cn}$ )

${ }^{1}$ Jilin Provincial Key Laboratory on Molecular and Chemical Genetics, The Second Hospital of Jilin University, 130041 Changchun, Jilin, China

Edited by D. Aberdam
}

- How does leptin act as a neurotrophic factor and exert neuroprotective actions in depression?

- What role does metabolism play in the physiological and pathological changes of depression and other mental illnesses?

\section{Introduction}

Depression is a highly prevalent and devastating mood disorder worldwide that exacts a serious physical and psychological burden on patients and their families. Numerous genetic, endocrine, environmental factors, and the interactions between those factors, may affect the progression of depressive disorders and contribute to individual susceptibility ${ }^{1,2}$. However, the underlying etiology and pathophysiology of depression remains incompletely understood. Traditional theories regarding the pathological mechanisms of depression implicate the disruption of neurotrophic factor regulation, monoamine neurotransmission and hippocampal neurogenesis ${ }^{3,4}$. Clinical data indicate that currently available treatments for depression possess various limitations in therapeutic efficacy and onset time ${ }^{5,6}$. Typical antidepressants treatments take several weeks or even months to achieve 
remission of depressive symptoms. Serious side effects, including acute nausea, sexual dysfunction, and weight gain, limit treatment efficacy ${ }^{7,8}$. Thus, identification of novel therapeutic targets based upon newer understanding of the etiology of affective disorders would greatly improve treatment.

Leptin is a 167 amino acid polypeptide product of $\mathrm{OB}$ gene produced and secreted by white adipose tissue ${ }^{9}$. Leptin can cross the blood brain barrier (BBB) and binds to a specific leptin receptor $(\text { LepRb })^{10}$ that is distributed in several specific brain regions, including several hypothalamic and thalamic regions, the substantia nigra pars compacta and the cerebellum, with lower levels of expression in some cortical areas and other brain regions. Leptin is an established and critical regulator of feeding behavior and energy expenditure ${ }^{11}$, but the brain distribution of LepRb also suggests that it may be involved in other neural functions. Several basic studies suggest that leptin has antidepressant effects and might be a potential therapeutic target for depression. LepRb deficiency leads to memory and cognitive impairments that are accompanied by alterations in hippocampal synaptic plasticity ${ }^{12}$. Acute administration of leptin has been reported to have antidepressant and anxiolytic effects in mice ${ }^{13}$. In addition, clinical studies have shown that the expression of leptin mRNA and protein are positively associated with depression severity. In this review, we present the available evidence, and try to illuminate the potential cellular and molecular mechanisms of leptin's antidepressant actions and its potential therapeutic capacity in the treatment of depression.

\section{Mechanisms of neural plasticity in depression}

The term "neural plasticity" represents the ability of a neuron or the brain to adapt to external or internal stimuli, involving a series of cellular events, including neurogenesis, cell migration, cell survival, synaptogenesis, and the modification of mature synapses ${ }^{14-16}$. Although such changes are generally adaptive, they may also be maladaptive under some conditions. Adaptive neuroplasticity in response to stimuli that evoke depressive symptomatology, including chronic stress, include both functional and structural changes, detected as neuroanatomical, neurochemical, and electrophysiological alterations ${ }^{17}$. Long-term exposure to stress and depression can result in neuronal atrophy, dendrite remodeling and synaptic dysfunctions in hippocampus, amygdala, and the prefrontal cortex (PFC) as shown in both in human and animal studies. Typical antidepressant drugs partly lead to reversal of stress-induced alterations in neuroplasticity ${ }^{15}$. This evidence provides support for the neuroplasticity hypothesis of depression. Regulation of neural plasticity appears to be involved in the therapeutic mechanisms of available antidepressant therapies, but more work remains to be done to demonstrate the necessity and importance of these changes.

\section{Dysfunction of neuronal morphology in depression}

Long-lasting alterations in cellular morphology, affecting cellular function and plasticity, may contribute to depression and other mood disorders ${ }^{18}$. Patients with major depressive disorder (MDD) have reduced neuronal density in the PFC, reduced synapse numbers, and reduced dendritic complexity compared to unaffected controls ${ }^{19}$. These changes in cellular morphology are consistent with the finding of structural atrophy in the hippocampus and PFC of depressed patients ${ }^{20,21}$. Clinical magnetic resonance imaging (MRI) studies show that patients with depression have reduced hippocampal volumes ${ }^{22-24}$, although this is not universally found ${ }^{25}$. Gerritsen et al. suggested that the amygdala:hippocampus volume ratio, rather than hippocampus or amygdala volume along is a better indicator of depression risk ${ }^{26}$. Moreover, there are positive associations between clinical improvement of depressive symptoms and hippocampal volume ${ }^{27}$. Additionally, depressive behavioral deficits induced by chronic stress correlate with the reduced number, and loss of function, of spine synapses in the $\mathrm{PFC}^{28}$. Indeed, rodent studies show that, exposure to stress results in a series of morphological changes, including neuronal atrophy and $\operatorname{loss}^{29,30}$. Taken together, these human and rodent studies indicate that depression has some similarities to neurodegenerative diseases that involve neuronal and structural atrophy $^{31}$. It is therefore logical to speculate that approaches that counter neuronal atrophy in the PFC and hippocampus might be potentially effective antidepressant treatments ${ }^{19,29}$. Future studies could target restoration of neural impairments and neuronal atrophy. The exact relationship between specific morphological changes and symptoms remains to be elucidated, however. These structural brain alterations might be neuroanatomical markers of current depressive state, and reversible, or predisposing factors that predate the onset of depression and be relatively difficult to reverse ${ }^{22,26}$. Determining the exact role of changes in synaptic plasticity in depression, and responses to antidepressant treatments, is an important goal of ongoing research.

\section{Synaptic plasticity and rapid-acting antidepressant}

With this in mind, the discovery of a rapid-acting antidepressants offers new insights into the pathogenesis of depression and the role of altered neuroplasticity in antidepressant effects. Ketamine is an N-methyl-D-aspartate (NMDA) receptor antagonist and widely used as a surgical anesthetic ${ }^{32}$. Ketamine exerts a rapid antidepressant effect in refractory depression patients (those responding poorly to traditional antidepressants). Berman and colleagues found that a low-dose of ketamine could 
exert antidepressant effects within hours that startlingly persisted for several months ${ }^{33}$. Basic science studies in animal models showed that the rapid antidepressant actions of ketamine might correlate with stimulation of synaptic structural plasticity and synaptogenesis ${ }^{29,34}$.

Synaptic plasticity, the most important and basic functional form of neuroplasticity, is acknowledged to play a fundamental role in the neurological pathogenesis of several neuropsychiatric diseases ${ }^{35,36}$. Basic science studies have focused on the underlying cellular and molecular mechanisms of ketamine's rapid-acting antidepressant effects. Ketamine blocks NMDA receptors located on inhibitory gamma-aminobutyric acid (GABA)ergic interneurons, resulting in a disinhibition of pyramidal cells of hippocampus ${ }^{37}$. Consequent release of glutamate activates the $\alpha$-amino-3-hydroxy-5-methyl-4isoxazolepropionic acid (AMPA) receptors, resulting in depolarization, influx of calcium, and stimulation of postsynaptic neurons ${ }^{38}$. Notably, the depolarization and calcium influx stimulate the release of the brain-derived neurotrophic factor (BDNF), which subsequently activates the Tropomyosin receptor kinase B (TrkB) receptor, and the mammalian target of rapamycin complex 1 (mTORC1) signaling pathway. mTORC1 can potentiate the synthesis of synaptic proteins are important for synaptogenesis and spinogenesis ${ }^{39,40}$. Since the treatment with the selective mTORC1 inhibitor rapamycin can block ketamine's rapid antidepressant actions, this establishes a critical regulatory role of mTORC1 in these effects ${ }^{41}$. Altered synaptic plasticity produced by antagonism of the glutamate receptor NMDA subtype on GABAergic interneurons is a likely mechanism for ketamine's rapid-acting antidepressant effects, but more work is needed to further confirm the adaptive significance of synaptic plasticity in all of these contexts: in response to chronic stress or resulting from other factors contributing to depression, as well as traditional and fast-acting antidepressants.

\section{The impact of BDNF on neuroplasticity in depression}

The classic neurotrophic hypothesis of depression presents that depression risk factors and antidepressant treatments can affect the development of depression, respectively, via decreasing or increasing the expression of neurotrophic factors, in particular $\mathrm{BDNF}^{3}$. BDNF plays a significant modulatory role in neuroplasticity, neurogenesis, and neuroprotection, and is the most widely reported neurotrophic factor implicated in pathogenesis of depression $^{42,43}$. In acute and chronic stress models, rodents exhibit decreased expression of both BDNF mRNA and protein in the PFC and hippocampus ${ }^{44}$. Antidepressant treatments, including SSRIs, and nonspecific monoamine reuptake inhibitors, reverse the reduction of hippocampal BDNF which accompanies chronic stress, resulting in reduced depressive behaviors $^{43}$. Clinical research has shown that blood BDNF levels negatively correlate with the severity of depressive symptoms $^{45,46}$.

Recent studies investigating changes in neural plasticity associated with stress and depression suggested that BDNF might contribute to functional and structural adaptions of synapses in depression-related brain regions. As mentioned above, BDNF activates TrkB receptors and induce activation of downstream phosphoinositide 3kinase (PI3K)/Akt and mitogen-activated protein kinases (MAPK)/ERK signaling pathways, both of which target the mTORC1 signaling pathway ${ }^{47}$. These pathways regulate several neuronal functions, including synaptic protein synthesis contributing to the formation and development of synapses ${ }^{48}$. Long-lasting stress can lead to synaptic loss, reduction of dendrite spine density, neuronal atrophy and apoptosis, all of which are related to inhibition of BDNF signaling pathways ${ }^{48}$. Consistent with that finding, it was found that heterozygous BDNF knockout rats, with reduced BDNF expression, display greater stress-induced decreases in spine density and dendrite length in the hippocampus and PFC. ${ }^{49}$. These findings suggest that BDNF expression protects against stress-induced deficits in neuroplasticity that are involved in depression and that differences in BDNF expression might underlie some part of individual differences in resilience and vulnerability to stress-induced psychiatric disorders. Thus, there might be an etiological link between altered BDNF and impaired neuroplasticity in depression. Moreover, typical antidepressants fail to stimulate glutamate release rapidly and subsequent activation of BDNF-TrkB signaling, which may contribute to the low efficacy of these drugs ${ }^{31}$. Thus, differences in this response, between individuals, as well as between treatments (e.g. traditional versus fast-acting antidepressants), might contributed to therapeutic efficacy.

\section{Leptin and depression \\ Human studies}

As summarized in Table 1, a number of clinical studies have investigated the link between leptin and depression. However, these studies investigating the relationship of MDD and leptin levels have yielded somewhat discrepant results. Initial studies reported lower leptin levels in depressive patients compared to healthy controls, which coincided with initial reports of leptin's antidepressants effects in animal models of depression ${ }^{50-53}$. However, higher leptin levels in MDD have also been reported (see Table 1).

Depressive patient subjects in clinical studies differ in selection criteria and recruitment strategies, which result in differences in study populations that could confound studies, such as those discussed above. Potential 
Table 1 Overview of measurements of leptin function in depression

\begin{tabular}{|c|c|c|c|c|c|c|}
\hline Subjects & $\begin{array}{l}\text { Sex }(F / \\
\mathrm{M}), N \%\end{array}$ & BMI & Assessment & $\begin{array}{l}\text { Source and } \\
\text { assay }\end{array}$ & Main findings & Refs. \\
\hline Control & $\begin{array}{l}301 / 196 \\
60.6\end{array}$ & $25.1 \pm 4.5$ & \multirow[t]{3}{*}{ DSM-IV } & \multirow[t]{3}{*}{$\begin{array}{l}\text { Fasting } \\
\text { blood; ELISA }\end{array}$} & \multirow[t]{3}{*}{$\begin{array}{l}\text { Higher leptin was associated with the atypical MDD } \\
\text { subtype both for remitted and current patients }\end{array}$} & \multirow[t]{3}{*}{60} \\
\hline Remitted MDD & $\begin{array}{l}480 / 231 \\
71.0\end{array}$ & $25.8 \pm 4.9$ & & & & \\
\hline Current MDD & $\begin{array}{l}754 / 308 \\
67.5\end{array}$ & $25.8 \pm 5.3$ & & & & \\
\hline Control & $16 / 26$ & $25.61 \pm 3.50$ & \multirow[t]{3}{*}{ HDRS } & \multirow{3}{*}{$\begin{array}{l}\text { Fasting } \\
\text { serum, EIISA }\end{array}$} & \multirow{3}{*}{$\begin{array}{l}\text { Higher leptin was found in patients with ADD than in } \\
\text { controls, but not in patients with DD-NA }\end{array}$} & \multirow[t]{3}{*}{59} \\
\hline ADD & $16 / 26$ & $26.36 \pm 4.17$ & & & & \\
\hline DD-NA & $20 / 31$ & $24.41 \pm 3.43$ & & & & \\
\hline $\begin{array}{l}\text { Minimal to no } \\
\text { depression }\end{array}$ & 60 & $29 \pm 7$ & $\begin{array}{l}\text { BDI-II scores } \\
0-13\end{array}$ & \multirow[t]{3}{*}{$\begin{array}{l}\text { Fasting } \\
\text { serum, ElISA }\end{array}$} & \multirow{3}{*}{$\begin{array}{l}\text { Leptin levels increased with the increasing BDI-II score; } \\
\text { participants with moderate to severe depression had the } \\
\text { highest levels of leptin }\end{array}$} & \multirow[t]{3}{*}{62} \\
\hline Mild depression & 67 & $31 \pm 9$ & $14-19$ & & & \\
\hline $\begin{array}{l}\text { Moderate to severe } \\
\text { depression }\end{array}$ & 63 & $33 \pm 8$ & $20-28$ & & & \\
\hline Control & 44 & $24.2 \pm 3.7$ & \multirow[t]{4}{*}{ DSM-IV } & \multirow{4}{*}{$\begin{array}{l}\text { Fasting } \\
\text { plasma, ELISA }\end{array}$} & \multirow{4}{*}{$\begin{array}{l}\text { Women with melancholic depression had higher leptin } \\
\text { levels than controls; no changes were observed between } \\
\text { undifferentiated or atypical patients and controls }\end{array}$} & \multirow[t]{4}{*}{61} \\
\hline Undifferentiated & 22 & $27.2 \pm 6.2$ & & & & \\
\hline Atypical & 16 & $28.9 \pm 7.1$ & & & & \\
\hline Melancholic & 51 & $25.8 \pm 6.0$ & & & & \\
\hline Control & $26 / 25$ & $16.9 \pm 2.0$ & \multirow[t]{3}{*}{ DSM-IV,BDI,BPRS } & \multirow{3}{*}{$\begin{array}{l}\text { Fasting } \\
\text { plasma, ELISA }\end{array}$} & \multirow{3}{*}{$\begin{array}{l}\text { Lower leptin and cholesterol levels in patients with MMD } \\
\text { than controls. }\end{array}$} & \multirow[t]{3}{*}{53} \\
\hline Schizophrenia & $39 / 39$ & $24.1 \pm 2.8$ & & & & \\
\hline MMD & $35 / 34$ & $20.6 \pm 2.7$ & & & & \\
\hline Healthy & 9 & $23.43 \pm 3.33$ & \multirow[t]{4}{*}{ DSM-IV } & \multirow{4}{*}{$\begin{array}{l}\text { Fasting } \\
\text { serum, ElISA }\end{array}$} & \multirow{4}{*}{$\begin{array}{l}\text { Women had higher leptin levels than men in both } \\
\text { depressive and healthy subjects; female patients had } \\
\text { significantly higher leptin levels compared to the control } \\
\text { females both before and after the treatment }\end{array}$} & \multirow[t]{4}{*}{57} \\
\hline Healthy female & 14 & $26.13 \pm 2.56$ & & & & \\
\hline Male MMD patients & 12 & $23.92 \pm 3.58$ & & & & \\
\hline Female MMD patients & 20 & $26.74 \pm 4.88$ & & & & \\
\hline Control & $\begin{array}{l}69 / 34 \\
67\end{array}$ & $26.0 \pm 5.5$ & \multirow[t]{2}{*}{ DSM-IV } & \multirow[t]{2}{*}{$\begin{array}{l}\text { Fasting } \\
\text { serum, ElISA }\end{array}$} & \multirow[t]{2}{*}{$\begin{array}{l}\text { Patients with } \mathrm{BD} \text { present lower leptin levels than those } \\
\text { with } \mathrm{MD} \text { and the control group. }\end{array}$} & \multirow[t]{2}{*}{101} \\
\hline Current depression & $\begin{array}{l}69 / 34 \\
67\end{array}$ & $26.5 \pm 4.9$ & & & & \\
\hline
\end{tabular}

$M D D$ major depressive disorder, $A D D$ depressive disorder with atypical features, $D D-N A$ depressive disorder without atypical features, $U D$ unipolar depression, $B D$ bipolar depression

confounding factors include differences in age, gender ratios, fat content and other metabolic factors, medication history, and subtypes of depression. The gender ratio of depressive and healthy subjects is a key factor that may address studies examining the role of leptin in depression. Female serum leptin concentrations are higher than men under normal physiological conditions ${ }^{54}$, and females also have higher rates of depression than males ${ }^{55,56}$. Esel and colleagues reported that female patients with MDD exhibited higher serum leptin levels than non-depressed females, while males had lower levels with or without a diagnosis of depression, which had no effect on leptin levels in males ${ }^{57}$ Therefore, the proportion of female participants in depressive and healthy subjects within a study could affect the observation of differences when investigating the association between leptin and depression.

Depression is a mood disorder with complicated etiopathogenesis, with different subtypes (or variable endophenotypes) which differ across individual patients. This complexity is in addition to such major divisions among mood disorders as MDD, bipolar disorder and seasonal 
affective disorder ${ }^{58}$, Gecici and colleagues first investigated the relationship between leptin and different clinical subtypes of MMD and found that depressive patients with atypical symptoms had higher serum leptin levels than $\operatorname{controls}^{59}$. No differences were found between depressive patients with a more typical profile and controls ${ }^{59}$. That leptin levels are higher in patients with atypical MDD, distinguished by significant weight gain and hyperphagia, was confirmed in a recent study ${ }^{60}$. Contradictory results exist, including a study showing that female patients with melancholic features have higher leptin levels than controls, but this study had a small sample size that may be influenced by the confounding factors previously mentioned $^{61}$. In any case, positive correlations between the severity of depression and leptin levels were observed. Serum leptin levels in patients with moderate to severe depression were higher than those with mild or minimal depression $^{62}$. Noting that those depressive patients with higher leptin levels also had elevated BMI, it is hard to conclude that leptin is positively linked to depression, rather than a compensatory effect induced by adiposity and metabolic syndrome.

A recent meta-analysis of studies comparing adipokines in MDD found no significant associations between peripheral leptin and MDD diagnosis ${ }^{63}$. Thus, based on the current results, leptin is not a biomarker for the diagnosis of depression or other mood disorders. In fact, the hyperleptinemia that has been observed in some cases of depression seems to associated more with central leptin resistance induced by adiposity and metabolic abnormities characterized by impaired leptin sensitivity. Given that mood disorders and metabolic syndromes co-occur and might interact with each other during their pathophysiological processes, increased leptin could be seen as an indirect proxy of metabolic abnormities attached to depression, rather than a pathological mediator. This could explain why elevated circulating leptin was detected most strongly in patients with atypical MDD.

\section{Animal studies}

As summarized in Table 2, several studies have examined stress-treated rodents or genetically modified rodents as depression models to investigate the link between leptin and depression. Initial studies found that exposure to chronic unpredictable stress (CUS) or chronic social defeat stress (CSDS) decreased leptin levels both in plasma and serum ${ }^{64,65}$. Leptin supplements, including peripheric leptin administration ${ }^{64,66}$ or transgenic overexpression of leptin ${ }^{67}$, could reverse behavioral deficits induced by chronic stress. These results indicate potential antidepressant actions of leptin, and implicate leptin deficiencies in the pathophysiological mechanisms of depression. Studies in mice with genetic deletions of the leptin gene and the LepRb gene further confirmed the critical role of leptin in depression. LepRb $(\mathrm{db} / \mathrm{db})$ mice $^{68,69}$ and Lep (ob/ob) mice ${ }^{67}$ display depression-like behavioral deficits (see Table 2). Leptin was reported to stimulate the glycogen synthase kinase (GSK)- $3 \beta / \beta$-catenin signaling pathway and reverse the inhibitory effects of a glucocorticoid receptor agonist on $\beta$-catenin, indicating that GSK-3 $\beta / \beta$-catenin signaling pathway-dependent neurogenesis is implicated in leptin's antidepressant effect $^{66}$. In addition, it is notable that leptin can affect the BDNF concentrations and subsequently the BDNF/TrkB signaling pathway. Based on much of the foregoing discussion, this would be a likely mechanism by which leptin might exert the antidepressant actions ${ }^{67}$.

\section{Role of leptin in neuroplasticity and depression Leptin and hippocampal synaptic plasticity}

Leptin was reported to modulate hippocampal transmission synaptic efficacy, including long-term potentiation (LTP) and long-term depression (LTD). Different doses of leptin, and other circulating hormones affected by leptin, affect LTP and LTD, but pharmacological studies investigating how leptin affects hippocampal synaptic function have shown inconsistent results. Administration of an intermediate dose of leptin directly into the hippocampus stimulates LTP and the hippocampal-dependent learning and memory; however, both higher and lower doses of leptin inhibit $\mathrm{LTP}^{71}$. Moult et al. found that leptin can reverse LTP at hippocampal CA1 synapses, in a time and concentrationdependent manner ${ }^{72}$. A competitive NMDA receptor antagonist can block this effect. Leptin-induced LTP preferentially occurs in the hippocampus of adult animal models. This may involve activation of GluN2A containing NMDA receptors and AMPA receptor trafficking $^{74}$. A recent study examined conditional knockout mice in which LepRb was ablated selectively in glutamatergic neurons in the hippocampus and PFC (LepRb cKO mice). This selective ablation of LepRb produced depression-like behavioral deficits and facilitation of NMDA receptor-induced LTD in the hippocampus. Interestingly, LepRb cKO mice are highly sensitive to Ro25-6981, an NMDA-type glutamate receptor subunit (GluN2B) antagonist, which produced antidepressant effects, but resistant to leptin. This finding suggests that leptin modulates excitatory synaptic strength via stimulating NMDA receptors ${ }^{73}$.

Leptin-induced facilitation of LTP is consistent with effects on learning and memory, but these may extend to other explain antidepressant effects of leptin. Glutamate dysfunction plays a major pathological role in depression, and other stress-related mood disorders ${ }^{75}$. Acute or chronic stress exposure increases glutamate release in limbic brain regions ${ }^{76}$. Adaptations to these stressinduced increases may connect stress to subsequent 


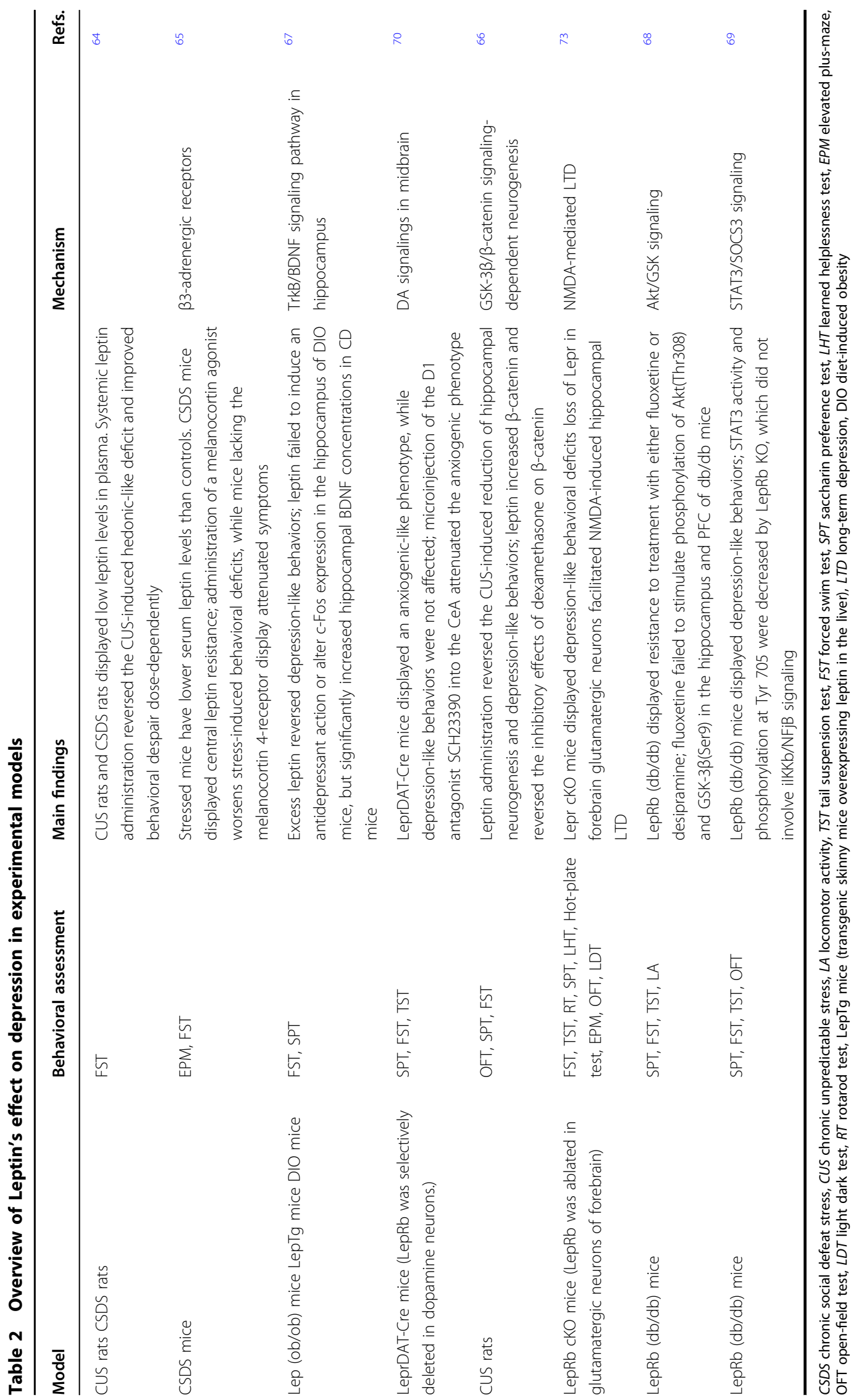




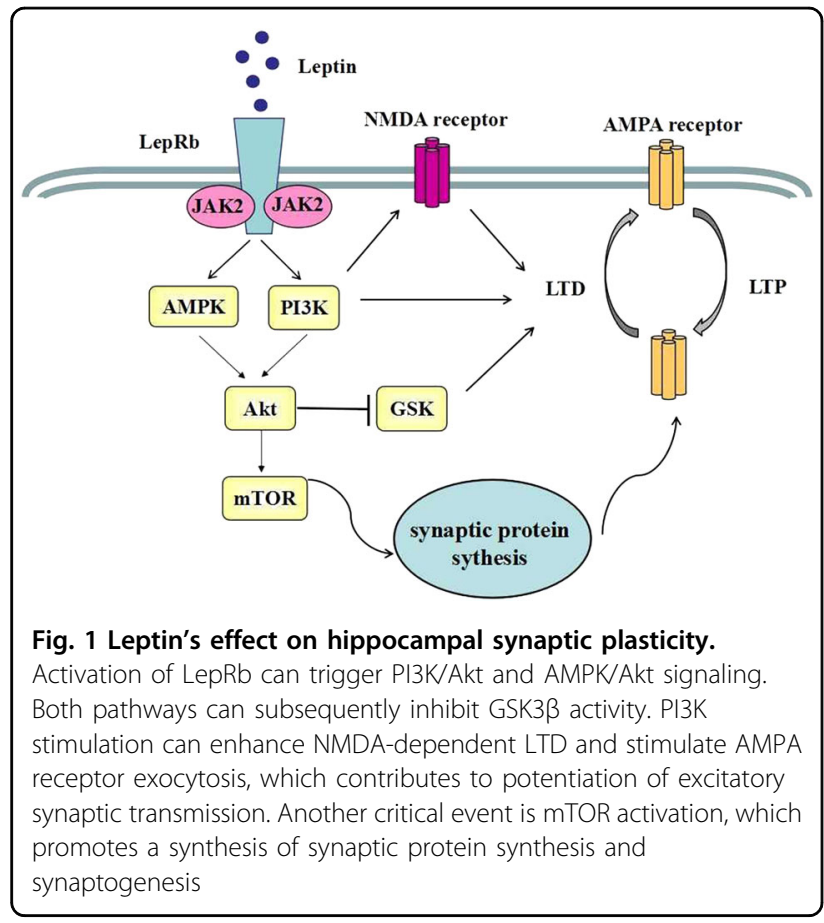

dysfunctional states, or amelioration of these states by antidepressant treatments. For example, tail suspension evokes a rapid increase in glutamate release in the hippocampal CA3 region and peripheral leptin injections decrease stress-evoked glutamate release ${ }^{77}$. Leptin' effect on glutamate may involve mTOR1 signaling, implicated in the effects of fast-acting antidepressants. mTOR plays a critical role in synaptic protein synthesis, and has been implicated in stress, depression, and antidepressant responses. Rapid-acting antidepressants stimulate in mTOR1 expression and facilitate mTORC1-dependent synaptic plasticity. Emerging evidence indicates that leptin can activate the PI3K/mTOR pathway in neuronal cells ${ }^{78}$. Activation of LepRb can stimulate mTOR activation and consequently promotes synthesis of synaptic proteins contributing to the maturation of old synapses and the formation of new synapses ${ }^{16}$ (see Fig. 1).

\section{Leptin and neurogenesis}

In addition to the data implicating leptin in synaptic neuroplasticity, accumulating evidence also shows that leptin plays a critical role in neurogenesis. This includes stimulating synaptogenesis and neurogenesis in the hypothalamus $^{79}$, but also extends to other brain regions where leptin induction of neurogenesis might underlie the antidepressant effect of leptin. Leptin can reverse the chronic stress-induced and glucocorticoid-induced inhibition of hippocampal neurogenesis and normalize behavioral deficits by activating the GSK-3 $\beta / \beta$-catenin signaling pathway ${ }^{66}$ (Fig. 2). Ser9 phosphorylation inhibits

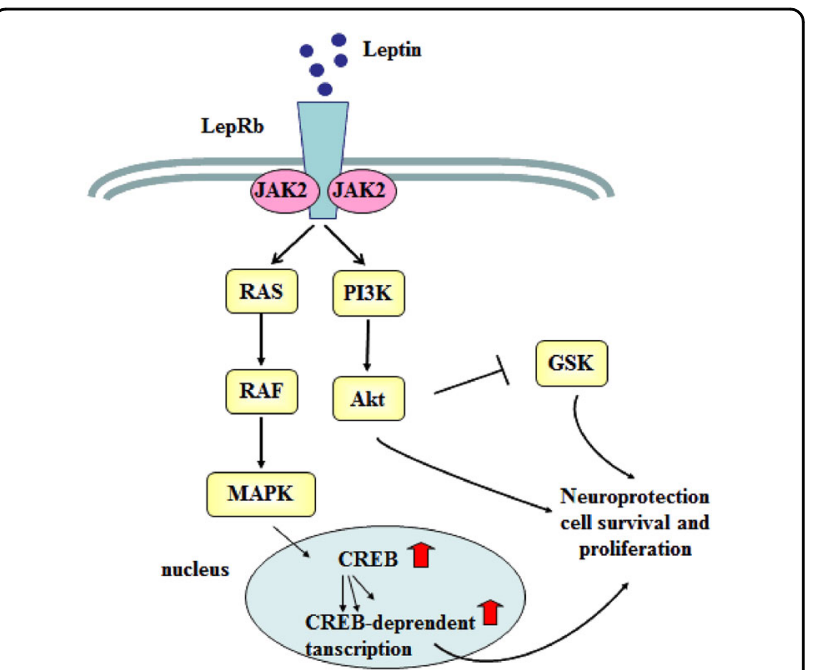

Fig. 2 Leptin activates multiple signaling pathways that potentiate neuroprotection, cell survival, and proliferation. Leptin binds to LepRb and activates the PI3K/Akt signaling pathway. Activating LepRb also stimulates the MAPK-signaling pathway. Both pathways promote neuroprotection, cell survival, and proliferation. The stimulation of PI3K/Akt decreases GSK-3 $\beta$ activity by phosphorylating GSK-3, promoting the expression of cell proliferation genes. Activation of the LepRb leads to CREB phosphorylation and initiation of CREB-dependent transcription, which also enhances cell proliferation genes. CREB CAMP response element-binding protein

the intracellular regulator GSK-3 $\beta$, while Tyr216 phosphorylation stimulates GSK- $3 \beta^{80}$. LepRb $(\mathrm{db} / \mathrm{db})$ mice have reduced Ser9 phosphorylation and a disinhibition of GSK-3 $\beta$ activity. Leptin inhibits GSK-3 $\beta$ activity via Ser9 phosphorylation in rats ${ }^{66}$. Leptin could inhibit GSK-3 $\beta$ activity and subsequently increase $\beta$-catenin signaling via activating the Akt signaling pathways ${ }^{69}$. Increased GSK$3 \beta / \beta$-catenin signaling might induce neuroprotection, cell survival, and proliferation ${ }^{81,82,83}$, which all relate to the proposed pathophysiological mechanisms of depression. In support of this conclusion, GSK-3 $\beta$ and $\beta$-catenin activity in the brain is associated with susceptibility to stress-related mood disorders ${ }^{84,85}$.

Previous studies reported that $\operatorname{LepRb}(\mathrm{db} / \mathrm{db})$ mice, which lack functional LepRb, have reduced spine density on dentate granule cells of the hippocampus ${ }^{86,87}$. Dhar et al. further suggested that leptin can stimulate functional dendrite spines and the formation of synapses in CA1 and CA3 neurons via the MAPK/ERK pathway and the subsequent induction of cAMP-response element binding protein (CREB)-dependent transcription ${ }^{88}$. CREB is an essential regulatory protein for cell survival, neuroprotection, and other neurotrophic factor-dependent synaptic events. That this signaling pathway is also regulated by leptin might indicate another mechanism by which leptin could regulate neurogenesis. Leptin also plays a significant role in the development of the cerebral 
cortex. LepRb mRNA exhibits high expression during cortical development and leptin deficiency could lead to a reduced number of cortical neurons during the early embryonic life ${ }^{89}$. It remains to be seen whether this also might implicate a role for leptin in cell survival in the adult cerebral cortex.

\section{Potential neurotrophic effects of leptin and BDNF}

Emerging evidence from human and animal studies demonstrates that leptin plays a potential neurotrophic role in the central nervous system (CNS). Leptin exerts neuroprotective actions and inhibits the dysfunction induced by a variety of neurotoxic insults, including accumulation of amyloid- $\beta$, exposure to excitotoxic glutamate, and ischemia ${ }^{90,91,92}$. Alhough the specific molecular mechanisms of leptin's neuroprotective effects are still obscure, the activation of downstream Jak2/STAT3, MAPK/ERK, PI3K/Akt, STAT5, and SHP-2/SOCS3 pathways might be involved. Leptin replacement therapy in leptin-deficient patients significantly increases the gray matter concentration in the anterior cingulate gyrus, cerebellum, and inferior parietal lobule ${ }^{93}$. This indicates that leptin either has somewhat broad effects on aspects of neural plasticity, neural proliferation, or neuroprotection. These effects have functional consequences; leptin supplement-induced structural changes detected in hippocampus and cortex improve cognition ${ }^{94}$.

Growing evidence demonstrates a link between BDNF expression and regulation of metabolic balance. Food deprivation, characterized by lower leptin secretion, can reduces BDNF expression within the dorsal vagal complex (DVC) and hippocampus ${ }^{95,96}$. Leptin stimulates BDNF mRNA translation in the dendrites of hypothalamic neurons ${ }^{97}$. Hippocampal BDNF expression is decreased in LepRb-deficient mice ${ }^{98}$. We have highlighted above that leptin can stimulate BDNF secretion via GSK-3 $\beta / \beta$-catenin and MAPK/ERK pathways. These results implicate leptin signaling in the synthesis and expression of BDNF, and consequently in BDNF-dependent functions, which includes depression and responses to antidepressant drugs.

Mice with diet-induced obesity have decreased hippocampal BDNF expression and behavior indicative of a depressive state ${ }^{67}$. Leptin treatment fails to increase hippocampal BDNF concentrations due to the impairment of leptin responses, which is known as leptin resistance induced by obesity ${ }^{67}$. The antidepressant effects of leptin thus appear to be mediated by activation of BDNF systems in hippocampus. These data further suggest that leptin responsiveness may be a biomarker, in some cases, of a depressive state associated with this mechanism. Moreover, reversal of leptin resistance by itself might restore normal BDNF function, and consequent effects on depressive states. Overall, these data implicate leptin and
BDNF in the regulation of energy homeostasis and depression, and suggest that there is substantial crosstalk between these physiological functions, which should not be surprising given some of the core symptoms of depression (that may include lethargy and changes in appetite). Leptin is therefore a neurotrophic factor, albeit perhaps an indirect one, although the specific cellular and molecular mechanisms underlying the effects of leptin discussed here, that are BDNF-dependent and BDNFindependent, require further investigation.

\section{Conclusion}

Neural plasticity is the capacity of the brain to undergo functional and/or structural adaptation in response to a range of intrinsic and extrinsic stimuli. The relationship between alterations in neural plasticity and depression, as well as the response to antidepressants, is not yet fully understood, but has been widely implicated by preclinical and clinical studies. Moreover, studies of fast-acting antidepressants are helping to illuminate the place of neural plasticity in depression and antidepressant effects. Ketamine rapidly increases the number of synaptic connections and reverses synapse loss resulting from chronic stress and depression. The emergence of ketamine as a novel antidepressant agent further supports the idea that synaptic plasticity is involved in the pathophysiology of depression and antidepressant responses. Leptin plays a vital role in neuroplasticity, especially in depressionrelated regions of the brain. Leptin can influence neuronal morphology and hippocampus synaptic transmission, and may act as a neurotrophic factor, if not directly, then indirectly via BDNF or other factors. Moreover, other neuropeptides, such as galanin, ghrelin, and corticotropin-releasing factor, have also been implicated in depression ${ }^{99,100}$. Consequently, leptin are potential therapeutic targets for treatment of depression, and elucidation of leptin-associated mechanisms underlying neural plasticity will enhance our understanding of the psychopathology of depression.

\section{Acknowledgements \\ This work was supported by grants from the Natural Science Foundation of China (NSFC) (81871070, 31471120, 31571126) and Jilin Science and \\ Technology Agency funding (Grant Nos. 20180519003JH and 20180414050GH).}

Conflict of interest

The authors declare that they have no conflict of interest.

Publisher's note

Springer Nature remains neutral with regard to jurisdictional claims in published maps and institutional affiliations.

Received: 15 January 2018 Revised: 2 June 2018 Accepted: 4 July 2018 Published online: 26 October 2018 


\section{References}

1. Hill, M. N., Hellemans, K. G., Verma, P., Gorzalka, B. B. \& Weinberg, J. Neurobiology of chronic mild stress: parallels to major depression. Neurosci. Biobehav. Rev. 36, 2085-2117 (2012).

2. Christoffel, D. J., Golden, S. A. \& Russo, S. J. Structural and synaptic plasticity in stress-related disorders. Rev. Neurosci. 22, 535-549 (2011).

3. Duman, R. S. \& Li, N. A neurotrophic hypothesis of depression: role of synaptogenesis in the actions of NMDA receptor antagonists. Philos. Trans. $R$. Soc. Lond. B Biol Sci. 367, 2475-2484 (2012).

4. Gardner, A. \& Boles, R. G. Beyond the serotonin hypothesis: mitochondria inflammation and neurodegeneration in major depression and affective spectrum disorders. Prog. Neuropsychopharmacol. Biol. Psychiatry 35, 730-743 (2011).

5. Irving, A. J. \& Harvey, J. Leptin regulation of hippocampal synaptic function in health and disease. Philos. Trans. R. Soc. Lond. B Biol. Sci. 369, 20130155 (2014).

6. Sestan-Pesa, M. \& Horvath, T. L. Metabolism and mental illness. Trends Mol Med. 22, 174-183 (2016)

7. Abdallah, C. G. et al. Ketamine's mechanism of action: a path to rapid-acting antidepressants. Depress. Anxiety 33, 689-697 (2016).

8. Fava, M. Weight gain and antidepressants. J. Clin. Psychiatry 61(Suppl. 11), 37-41 (2000).

9. Otero, M. et al. Leptin: a metabolic hormone that functions like a proinflammatory adipokine. Drug News Perspect. 19, 21-26 (2006)

10. Tartaglia, L. A. et al. Identification and expression cloning of a leptin receptor, OB-R. Cell 83, 1263-1271 (1995).

11. Halaas, J. L. et al. Weight-reducing effects of the plasma protein encoded by the obese gene. Science 269, 543-546 (1995).

12. Shanley, L. J., Irving, A. J. \& Harvey, J. Leptin enhances NMDA receptor function and modulates hippocampal synaptic plasticity. J. Neurosci. 21 RC186 (2001).

13. Liu, J. et al. Acute administration of leptin produces anxiolytic-like effects: a comparison with fluoxetine. Psychopharmacology 207, 535-545 (2010).

14. Pilar-Cuéllar, F. et al. Neural plasticity and proliferation in the generation of antidepressant effects: hippocampal implication. Neural Plast. 2013, 537265 (2013).

15. Liu, W. et al. The role of neural plasticity in depression: from hippocampus to prefrontal cortex. Neural Plast. 2017, 6871089 (2017).

16. Duman, R. S., Aghajanian, G. K., Sanacora, G. \& Krystal, J. H. Synaptic plasticity and depression: new insights from stress and rapid-acting antidepressants. Nat Med. 22, 238-249 (2016).

17. Fadel, J. R., Jolivalt, C. G. \& Reagan, L. P. Food for thought: the role of appetitive peptides in age-related cognitive decline. Ageing Res. Rev. 12 764-776 (2013).

18. Roozendaal, B., McEwen, B. S. \& Chattarji, S. Stress, memory and the amygdala. Nat Rev. Neurosci. 10, 423-433 (2009).

19. Rajkowska, G. et al. Morphometric evidence for neuronal and glial prefrontal cell pathology in major depression. Biol Psychiatry 45, 1085-1098 (1999).

20. Bearden, C. E. et al. Altered hippocampal morphology in unmedicated patients with major depressive illness. ASN Neuro 1, e00020 (2009).

21. Stratmann, M. et al. Insular and hippocampal gray matter volume reductions in patients with major depressive disorder. PLOS ONE 9, e102692 (2014).

22. Janssen, J. et al. Hippocampal volume and subcortical white matter lesions in late life depression: comparison of early and late onset depression. J. Neurol. Neurosurg. Psychiatry 78, 638-640 (2007)

23. Ahdidan, J. et al. Longitudinal MR study of brain structure and hippocampus volume in major depressive disorder. Acta Psychiatr. Scand. 123, 211-219 (2011).

24. Saylam, C., Uçerler, H., Kitiş, O., Ozand, E. \& Gönül, A. S. Reduced hippocampal volume in drug-free depressed patients. Surg. Radiol. Anat. 28, 82-87 (2006).

25. Eker, M. C. et al. Smaller hippocampus volume is associated with short variant of 5-HTTLPR polymorphism in medication-free major depressive disorder patients. Neuropsychobiology 63, 22-28 (2011).

26. Gerritsen, L. et al. Amygdala to hippocampal volume ratio is associated with negative memory bias in healthy subjects. Psychol. Med. 42, 335-343 (2012)

27. Schuch, F. B. et al. Neurobiological effects of exercise on major depressive disorder: a systematic review. Neurosci. Biobehav. Rev. 61, 1-11 (2016).

28. Shansky, R. M. \& Morrison, J. H. Stress-induced dendritic remodeling in the medial prefrontal cortex: effects of circuit, hormones and rest. Brain Res. 1293, 108-113 (2009).

29. Duman, R. S. \& Aghajanian, G. K. Synaptic dysfunction in depression: potential therapeutic targets. Science 338, 68-72 (2012).
30. McEwen, B. S., Eiland, L., Hunter, R. G. \& Miller, M. M. Stress and anxiety: structural plasticity and epigenetic regulation as a consequence of stress. Neuropharmacology 62, 3-12 (2012).

31. Gerhard, D. M., Wohleb, E. S. \& Duman, R. S. Emerging treatment mechanisms for depression: focus on glutamate and synaptic plasticity. Drug Discov. Today 21, 454-464 (2016).

32. du, J. K. G. et al. Potential involvement of serotonergic signaling in ketamine's antidepressant actions: a critical review. Prog. Neuropsychopharmacol. Biol Psychiatry 71, 27-38 (2016).

33. Berman, R. M. et al. Antidepressant effects of ketamine in depressed patients. Biol. Psychiatry 47, 351-354 (2000)

34. Strasburger, S. E. et al. What is the mechanism of Ketamine's rapid-onset antidepressant effect? A concise overview of the surprisingly large number of possibilities. J. Clin. Pharm. Ther. 42, 147-154 (2017).

35. Nanou, E. \& Catterall, W. A. Calcium channels, synaptic plasticity, and neuropsychiatric disease. Neuron 98, 466-481 (2018).

36. Pilato, F. et al. Synaptic plasticity in neurodegenerative diseases evaluated and modulated by in vivo neurophysiological techniques. Mol. Neurobiol. 46, 563-571 (2012).

37. Deutschenbaur, L. et al. Role of calcium, glutamate and NMDA in major depression and therapeutic application. Prog. Neuropsychopharmacol. Biol Psychiatry 64, 325-333 (2016)

38. Tizabi, Y., Bhatti, B. H., Manaye, K. F., Das, J. R. \& Akinfiresoye, L. Antidepressantlike effects of low ketamine dose is associated with increased hippocampal AMPA/NMDA receptor density ratio in female Wistar-Kyoto rats. Neuroscience 213, 72-80 (2012)

39. Li, N. et al. mTOR-dependent synapse formation underlies the rapid antidepressant effects of NMDA antagonists. Science 329, 959-964 (2010).

40. Williams, N. R. \& Schatzberg, A. F. NMDA antagonist treatment of depression. Curr. Opin. Neurobiol. 36, 112-117 (2016).

41. Harraz, M. M. \& Snyder, S. H. Antidepressant actions of ketamine mediated by the mechanistic target of rapamycin, nitric oxide, and rheb. Neurotherapeutics 14, 728-733 (2017)

42. Manju, S., Vignoli, B., Canossa, M. \& Blum, R. Neurobiology of local and intercellular BDNF signaling. Pflugers Arch. 469, 593-610 (2017).

43. Begni, V., Riva, M. A. \& Cattaneo, A. Cellular and molecular mechanisms of the brain-derived neurotrophic factor in physiological and pathological conditions. Clin. Sci. 131, 123-138 (2017).

44. Grønli, J. et al. Chronic mild stress inhibits BDNF protein expression and CREB activation in the dentate gyrus but not in the hippocampus proper. Phar macol. Biochem. Behav. 85, 842-849 (2006).

45. Lee, B. H., Kim, H., Park, S. H. \& Kim, Y. K. Decreased plasma BDNF level in depressive patients. J. Affect. Disord. 101, 239-244 (2007).

46. Aydemir, C. et al. Brain-derived neurotrophic factor (BDNF) changes in the serum of depressed women. Prog. Neuropsychopharmacol. Biol. Psychiatry 30, 1256-1260 (2006).

47. Zhang, J. C., Yao, W. \& Hashimoto, K. Brain-derived neurotrophic factor (BDNF)-TrkB signaling in inflammation-related depression and potential therapeutic targets. Curr. Neuropharmacol. 14, 721-731 (2016).

48. Liu, W. X. et al. Regulation of glutamate transporter 1 via BDNF-TrkB signaling plays a role in the anti-apoptotic and antidepressant effects of ketamine in chronic unpredictable stress model of depression. Psychopharmacology $\mathbf{2 3 3}$ 405-415 (2016).

49. Magariños, A. M. et al. Effect of brain-derived neurotrophic factor haploinsufficiency on stress-induced remodeling of hippocampal neurons. Hippocampus 21, 253-264 (2011).

50. Kraus, T., Haack, M., Schuld, A., Hinze-Selch, D. \& Pollmächer, T. Low leptin levels but normal body mass indices in patients with depression or schizophrenia. Neuroendocrinology 73, 243-247 (2001).

51. Yang, $K$ et al. Levels of serum interleukin (IL)-6, IL-1beta, tumour necrosis factor-alpha and leptin and their correlation in depression. Aust. N. Z. J. Psychiatry 41, 266-273 (2007).

52. Eikelis, N. et al. Reduced brain leptin in patients with major depressive disorder and in suicide victims. Mol. Psychiatry 11, 800-801 (2006).

53. Jow, G. M., Yang, T. T. \& Chen, C. L. Leptin and cholesterol levels are low in major depressive disorder, but high in schizophrenia. J. Affect. Disord. 90, 21-27 (2006).

54. Ma, Z. et al. Radioimmunoassay of leptin in human plasma. Clin. Chem. 42 942-946 (1996). 
55. Hankin, B. L. et al. Development of depression from preadolescence to young adulthood: emerging gender differences in a 10-year longitudinal study. J. Abnorm. Psychol. 107, 128-140 (1998).

56. Sowa, C. J. \& Lustman, P. J. Gender differences in rating stressful events, depression, and depressive cognition. J. Clin. Psychol. 40, 1334-1337 (1984).

57. Esel, E. et al. Effects of antidepressant treatment and of gender on serum leptin levels in patients with major depression. Prog. Neuropsychopharmacol. Biol Psychiatry 29, 565-570 (2005).

58. Henter, I. D. et al. Mood therapeutics: novel pharmacological approaches for treating depression. Expert Rev. Clin. Pharmacol. 10, 153-166 (2017).

59. Gecici, O. et al. High serum leptin levels in depressive disorders with atypical features. Psychiatry Clin. Neurosci. 59, 736-738 (2005).

60. Milaneschi, Y., Lamers, F., Bot, M., Drent, M. L. \& Penninx, B. W. Leptin dysregulation is specifically associated with major depression with atypical features: evidence for a mechanism connecting obesity and depression. Biol Psychiatry 81, 807-814 (2017).

61. Cizza, G. et al. Clinical subtypes of depression are associated with specific metabolic parameters and circadian endocrine profiles in women: the power study. PLOS ONE 7, e28912 (2012).

62. Morris, A. A. et al. The association between depression and leptin is mediated by adiposity. Psychosom. Med. 74, 483-488 (2012).

63. Carvalho, A. F. et al. Adipokines as emerging depression biomarkers: a systematic review and meta-analysis. J. Psychiatr. Res. 59, 28-37 (2014).

64. Lu, X. Y., Kim, C. S., Frazer, A. \& Zhang, W. Leptin: a potential novel antidepressant. Proc. Natl Acad. Sci. USA 103, 1593-1598 (2006).

65. Chuang, J. C. et al. A beta3-adrenergic-leptin-melanocortin circuit regulates behavioral and metabolic changes induced by chronic stress. Biol. Psychiatry 67, 1075-1082 (2010).

66. Garza, J. C., Guo, M., Zhang, W. \& Lu, X. Y. Leptin restores adult hippocampa neurogenesis in a chronic unpredictable stress model of depression and reverses glucocorticoid-induced inhibition of GSK-3 $\beta / \beta$-catenin signaling Mol Psychiatry 17, 790-808 (2012).

67. Yamada, N. et al. Impaired CNS leptin action is implicated in depression associated with obesity. Endocrinology 152, 2634-2643 (2011).

68. Guo, M. \& Lu, X. Y. Leptin receptor deficiency confers resistance to behavioral effects of fluoxetine and desipramine via separable substrates. Transl. Psychiatry 4, e486 (2014).

69. Liu, W. et al. Leptin receptor knockout-induced depression-like behaviors and attenuated antidepressant effects of exercise are associated with STAT3/ SOCS3 signaling. Brain Behav. Immun. 61, 297-305 (2017)

70. Liu, J., Perez, S. M., Zhang, W., Lodge, D. J. \& Lu, X. Y. Selective deletion of the leptin receptor in dopamine neurons produces anxiogenic-like behavior and increases dopaminergic activity in amygdala. Mol Psychiatry 16, 1024-1038 (2011).

71. Wayner, M. J., Armstrong, D. L., Phelix, C. F. \& Oomura, Y. Orexin-A (Hypocretin-1) and leptin enhance LTP in the dentate gyrus of rats in vivo. Peptides 25, 991-996 (2004)

72. Moult, P. R., Milojkovic, B. \& Harvey, J. Leptin reverses long-term potentiation at hippocampal CA1 synapses. J. Neurochem. 108, 685-696 (2009).

73. Guo, M. et al. Forebrain glutamatergic neurons mediate leptin action ondepression-like behaviors and synaptic depression. Transl. Psychiatry 2, e83 (2012).

74. Harvey, J. Leptin regulation of neuronal morphology and hippocampal synaptic function. Front. Synaptic Neurosci. 5, 3 (2013).

75. Gulyaeva, N. V. Interplay between brain BDNF and glutamatergic systems: a brief state of the evidence and association with the pathogenesis ofdepression. Biochemistry 82, 301-307 (2017).

76. Lowy, M. T., Wittenberg, L. \& Yamamoto, B. K. Effect of acute stress on hippocampal glutamate levels and spectrin proteolysis in young and aged rats. J. Neurochem. 65, 268-274 (1995).

77. Wang, X., Zhang, D. \& Lu, X. Y. Dentate gyrus-CA3 glutamate release/NMDA transmission mediates behavioral despair and antidepressant-like responses to leptin. Mol Psychiatry 20, 509-519 (2015).

78. Thon, M., Hosoi, T., Yoshii, M. \& Ozawa, K. Leptin induced GRP78 expression through the PI3K-mTOR pathway in neuronal cells. Sci. Rep. 4, 7096 (2014).

79. Bouret, S. G. Neurodevelopmental actions of leptin. Brain Res. 1350, 2-9 (2010).
80. Liu, L., Wan, W., Xia, S., Kalionis, B. \& Li, Y. Dysfunctional Wnt/ $\beta$-catenin signaling contributes to blood-brain barrier breakdown in Alzheimer's disease. Neurochem. Int. 75, 19-25 (2014).

81. Liu, X. H. et al. Blocking GSK3ß-mediated dynamin1 phosphorylation enhances BDNF-dependent TrkB endocytosis and the protective effects of BDNF in neuronal and mouse models of Alzheimer's disease. Neurobiol. Dis. 74, 377-391 (2015).

82. Joksimovic, M. \& Awatramani, R. Wnt/ $\beta$-catenin signaling in midbrain dopaminergic neuron specification and neurogenesis. J. Mol Cell Biol. 6 27-33 (2014).

83. Qu, Z. et al. Wnt/ß-catenin signalling pathway mediated aberrant hippocampal neurogenesis in kainic acid-induced epilepsy. Cell. Biochem. Funct. 35 472-476 (2017).

84. Dias, $C$. et al. $\beta$-catenin mediates stress resilience through Dicer1/microRNA regulation. Nature 516, 51-55 (2014).

85. Luykx, J. J. et al. The involvement of GSK3beta in bipolar disorder: integrating evidence from multiple types of genetic studies. Eur. Neuropsychopharmacol. 20, 357-368 (2010).

86. Stranahan, A. M. et al. Voluntary exercise and caloric restriction enhance hippocampal dendritic spine density and BDNF levels in diabetic mice. Hippocampus 19, 951-961 (2009).

87. Wosiski-Kuhn, M., Erion, J. R., Gomez-Sanchez, E. P., Gomez-Sanchez, C. E. \& Stranahan, A. M. Glucocorticoid receptor activation impairs hippocampal plasticity by suppressing BDNF expression in obese mice. Psychoneuroendocrinology 42, 165-177 (2014).

88. Dhar, M. et al. Leptin induces hippocampal synaptogenesis via CREBregulated microRNA-132 suppression of p250GAP. Mol. Endocrinol. 28, 1073-1087 (2014).

89. Cervero, A. et al. The role of the leptin in reproduction. Curr. Opin. Obstet. Gynecol. 18, 297-303 (2006).

90. Doherty, G. H., Beccano-Kelly, D., Yan, S. D., Gunn-Moore, F. J. \& Harvey, J. Leptin prevents hippocampal synaptic disruption and neuronal cell death induced by amyloid B. Neurobiol. Aging 34, 226-237 (2013).

91. Zhang, J. Y. et al. Leptin administration alleviates ischemic brain injury in mice by reducing oxidative stress and subsequent neuronal apoptosis. J. Trauma Acute Care Surg. 72, 982-991 (2012).

92. Zhang, F., Wang, S., Signore, A. P. \& Chen, J. Neuroprotective effects of leptin against ischemic injury induced by oxygen-glucose deprivation and transient cerebral ischemia. Stroke 38, 2329-2336 (2007).

93. London, E. D. et al. Short-term plasticity of gray matter associated with leptin deficiency and replacement. J. Clin. Endocrinol. Metab. 96, E1212-E1220 (2011).

94. Matochik, J. A. et al. Effect of leptin replacement on brain structure in genetically leptin-deficient adults. J. Clin. Endocrinol. Metab. 90, 2851-2854 (2005)

95. Bariohay, B., Lebrun, B., Moyse, E. \& Jean, A. Brain-derived neurotrophic factor plays a role as an anorexigenic factor in the dorsal vagal complex. Endocrinology 146, 5612-5620 (2005).

96. Iwasa, T. et al. Developmental changes in the hypothalamic mRNA expression levels of brain-derived neurotrophic factor and serum leptin levels: Their responses to fasting in male and female rats. Int. J. Dev. Neurosci. 54, 1-5 (2016).

97. Liao, G. Y. et al. Dendritically targeted Bdnf mRNA is essential for energy balance and response to leptin. Nat Med. 18, 564-571 (2012).

98. Stranahan, A. M., Arumugam, T. V. \& Mattson, M. P. Lowering corticosterone levels reinstates hippocampal brain-derived neurotropic factor and Trkb expression without influencing deficits in hypothalamic brain-derived neurotropic factor expression in leptin receptor-deficient mice. Neuroendocrinology 93, 58-64 (2011).

99. Madaan, V. \& Wilson, D. R. Neuropeptides: relevance in treatment of depression and anxiety disorders. Drug News Perspect. 22, 319-324 (2009).

100. Wittekind, D. A. \& Kluge, M. Ghrelin in psychiatric disorders-a review. Psychoneuroendocrinology 52, 176-194 (2015).

101. Cordas, G. et al. Leptin in depressive episodes: is there a difference between unipolar and bipolar depression. Neuroendocrinology 101, 82-86 (2015). 